\title{
Demonstration of Apparatus
}

\begin{tabular}{l|l|l}
\hline H.M. & Dekking $\dagger$ \\
\hline
\end{tabular}

(1) An Apparatus for Measuring Stereoscopic near Vision

If an object is placed before a concave mirror at a distance of $2 \mathrm{ft}$

the image lies at the same place. Small movements of the object along the optical axis result in movements of the image in opposite directions

and if one has good stereoscopic vision

an alignment of the two in the same plane can be made with great precision. A disturbing factor is the image of the surrounding world in the mirror. This can be eliminated by placing a black screen (at a distance of $2 \mathrm{ft}$ ) at the lower half of the field; the inverted image of this screen then fills exactly the upper half so that the whole field is black. One part of the test object (two white vertical lines) is put on the mirror side of the screen; its image floats in the air just above it. The other part (one black line) is situated on the observers' side of the screen. Displacement of the mirror is caused by a fine screw

and by turning this screw the single line comes exactly in the same plane as the two others.

(2) Dynamic Tonometry (published in Ophthalmologica 15A: 59-74

1967) 